\title{
On the Relationship Between Red Rectangle and Diffuse Interstellar Bands
}

\author{
Frédéric Zagury ${ }^{*}$
}

Institut Louis de Broglie, 23 rue Marsoulan, 75012 Paris, France

\begin{abstract}
A careful examination of Red Rectangle bands which have been considered as diffuse interstellar bands (DIBs) in emission shows that a few are likely to be artifacts in the spectrum. Some others result from atmospheric extinction. Consequences for the Red Rectangle band/DIB associations are examined.

I will also comment a striking resemblance between the DIB spectrum and the spectrum of $\mathrm{NO}_{2}$ in the $6150-6250 \AA$ region. This suggests that some DIBs could be provoked by atmospheric molecules.
\end{abstract}

Keywords: Atmospheric effects, ISM: lines and bands, planetary nebulae: general, planetary nebulae: individual (HD44179).

\section{INTRODUCTION}

The spectrum of the Red Rectangle nebula consists in a hierarchy of interwoven structures. The broad ERE (Extended Red Emission) bump, responsible for the red color of the nebula, extends over more than $2000 \AA$ between $5500 \AA$ and $7500 \AA$. On the bump, with a resolution of a few $\AA$, Schmidt et al. [1] distinguishes six separate sets of emission-like structures (see also [2]). An increase of the resolution by a factor of ten (a few $0.1 \AA$, [3]) reveals fine structure in the three largest Schmidt et al. bands and new features on the bump.

At the beginning of the 1990s' correlations were found $[4,5]$ between prominent Red Rectangle bands $(\lambda \lambda 5799$, 5855 in the $5800 \AA$ complex, $\lambda 6380, \lambda 6617)$, and the position in the spectrum of important DIBs $(\lambda \lambda 5797,5850$, 6379, 6614). [6, 7] added a few such Red Rectangle band/DIB associations. These relationships first suggested that the same carrier was seen in absorption in the direction of reddened stars and in emission in the Red Rectangle.

However, peak wavelengths of the Red Rectangle bands do not coincide with DIB wavelengths. For the red-degraded (with a steep blue edge and a long red tail) Red Rectangle bands, peak wavelengths are not at a fixed position. They progressively shift to the blue when distance from HD44179 (the star at the center of the Red Rectangle which illuminates the nebula) increases, due to a narrowing of their red wings $[3,6-8]$. Blue edges are stable and remain along the red side of the corresponding DIBs. It is thus the blue band head of a red-degraded Red Rectangle band that matches the corresponding DIB wavelength [9]. [3, 10] definitely proved that Red Rectangle bands' central wavelengths never reach DIB wavelengths.

For the symmetric $\lambda 6378$ Red Rectangle band, DIBs have been found on each of its sides ( $\lambda \lambda 6376$ and 6379) $[6,9]$.

The non-convergence of Red Rectangle bands to DIB wavelengths led [10] to question the DIB/Red Rectangle band

*Address for correspondence to this author at the Institut Louis de Broglie, 23 rue Marsoulan, 75012 Paris, France E-mail: fzagury@wanadoo.fr relationships. The very large number of DIBs known today (over 380 in the recent Hobbs et al. survey [11], against less than 80 at the time the first DIB/Red Rectangle band relationships were established), can indeed favor accidental coincidences.

The relationship, however, not only relies on wavelength positions, but also on physical parameters such as bands' strength and shape ([6,9] and references therein). The $5800 \AA$ region for instance contains among the most important DIBs and the most spectacular Red Rectangle bands, which of course accredits the idea of a relation between the two.

In two previous studies $[12,13]$ on the Red Rectangle I have outline the importance atmospheric extinction (more specifically: atmospheric absorption and starlight diffracted or scattered in the atmosphere) might have in the observed spectrum of the nebula. This past work may contribute to the discussion on the DIB/Red Rectangle band associations in two ways.

It should first be remarked that the positions of DIB wavelengths on the sides of Red Rectangle bands, if meaningful, characterize an absorption spectrum. In an absorption spectrum, it is the minima in the spectrum which correspond to absorption lines' positions and have physical meaning, as it is observed in the Red Rectangle nebula.

I have also found [13] that the sharp $\lambda 6378$ and the diffuse $\lambda 6225$ Schmidt et al. bands are present in the background of the Red Rectangle nebula observations. As mentioned above, $\lambda 6378$ is in-between two DIBs. According to table 5 in [3] correspondences can be found between substructures in $\lambda 6225$, and DIBs in the $\lambda 6200$ complex [14]. The presence of the two bands in background spectra either questions their relation to DIBs, or has implications which need to be investigated: can there be an atmospheric origin to some DIBs; what alternative is there?

In the present study, I will first re-examine a few Red Rectangle bands which have been associated to DIBs (Sect. 4; Sects. 2 and 3 present the data). Results of this analysis, the problems they raise and hypotheses they suggest, are discussed in Sect. 5. 
Table 1. Red Rectangle Observations

\begin{tabular}{|c|c|c|c|c|c|c|c|c|c|c|}
\hline & Observatory & Spectro. & Slit & $\Delta \Theta^{(2)}$ & $\Delta \lambda^{(3)}$ & $\lambda_{\text {inf }}-\lambda_{\text {sup }}{ }^{(4)}$ & $\mathbf{d}^{(5)}$ & Data $^{(6)}$ & Obs. date & Refs. $^{(9)}$ \\
\hline \hline 1 & F. L. Whipple & FAST & $3^{\prime \prime} \times 3^{\prime}$ & 1.2 & 1.4 & $3660-7530$ & 14 & - & 2001.12 .22 & {$[13]$} \\
\hline $2^{(7)}$ & La Silla Paranal & EMMI & $1^{\prime \prime} \times 3^{\prime}$ & 0.268 & 0.32 & $5540-6195$ & 6 & $01: 07: 07.550$ & 1998.01 .26 & {$[3,13]$} \\
\hline $3^{(8)}$ & La Silla Paranal & EMMI & $1^{\prime \prime} \times 3^{\prime}$ & 0.268 & 0.64 & $5520-6880$ & 11 & $01: 58: 37.630$ & 1998.01 .26 & {$[3,13]$} \\
\hline $4^{(8)}$ & La Silla Paranal & EMMI & $1^{\prime \prime} \times 3^{\prime}$ & 0.268 & 0.64 & $5520-6880$ & 11 & $03: 01: 24.630$ & 1998.01 .26 & {$[3,13]$} \\
\hline
\end{tabular}

(1) Slit dimensions on the sky.

(2) Spatial resolution $($ (").

(3) Spectral resolution $(\AA)$.

(4) Wavelength coverage.

(5) Distance (") from HD44179 (for all observations the slit is roughly perpendicular to the north-south axis of the nebula).

(6) Spectrum's designation in ESO archive. Should be preceded by 'ONTT.1998-0126T'.

(7) Cut C in [3], figure (1).

(8) Cut D, in [3], figure (1)

(9) Reference papers.

Different carriers are proposed for the DIBs but no laboratory spectrum which could support a concrete identification has ever been presented. In Sect. 5.4, I will comment on the striking resemblance I have found between the DIB spectrum in the $6200 \AA$ region (related to Red Rectangle $\lambda 6225$ diffuse band) and the spectrum of $\mathrm{NO}_{2}$.

\section{DATA}

Most of the observations used in this work come from $[12,13]$.

Red Rectangle data consist in three observations retrieved from the ESO Archive Facility, and one observation of the nebula observed at the Fred L. Whipple Observatory with the FAST spectrograph [15]. Main characteristics of these data are summarized in Table $\mathbf{1}$.

As in my previous articles, the spectrum at pixel ' $x$ ' in the 2-D array of one of these long-slit observations is noted ' $s \mathrm{x}$ '. Figures present raw data, flat-fielded but not corrected for atmospheric extinction and with no background subtracted.

A long-slit ESO data-set consists in 600 spectra. For the two observations $11^{\prime \prime}$ from HD44179 the nebula extends roughly from $s 330$ to $s 429\left(\sim 27^{\prime \prime}\right.$ on the sky, [13]). Maximum brightness in the nebula is reached for spectra close to $s 360$.

The FAST observation has 120 pixels. The nebula extends from $\sim s 38$ to $\sim s 63\left(\sim 31^{\prime \prime}\right)$.

In Sect. $5.4 \mathrm{I}$ will also use spectra with comparable resolutions of $\mathrm{BD}+40^{\circ} 4220(\mathrm{R}=45000, \Delta \lambda \sim 0.03 \AA$, [16]), provided by G. Galazutdinov, and of $\mathrm{NO}_{2}$ (laboratory spectrum at room temperature, pressure $\sim 10^{-3}$ torr, res. $=$ $0.01 \AA$ ), due to A. Jenouvrier (Université de Reims, France).

The latter spectrum is a private communication from A. Jenouvrier. It was initially designed for studies of the atmosphere and has served for the HITRAN (HIghresolution TRANsmission molecular absorption; http:// cfawww.harvard.edu/hitran/) database.

\section{RED RECTANGLE BANDS SELECTION}

Van Winckel et al. [3] (table 5) establish a link between a few Red Rectangle bands and DIBs. My purpose in this study is to re-analyse the origin of these bands. First column of Table 2 gives the peak wavelength of each band, second and third the estimated limits on each of their sides. $\lambda 6221$ (table 3 in [3]) was added, because of its importance in the $\lambda 6225$ complex (Sect. 4.4).

Table 2. DIBs' Related Red Rectangle Bands ${ }^{(1)}$

\begin{tabular}{|c|c|c|c|c|}
\hline$\lambda_{\mathrm{c}}^{(2)}$ & $\lambda_{\text {inf }}^{(3)}$ & $\lambda_{\text {sup }}^{(4)}$ & DIBs $^{(5)}$ & Origin $^{(6)}$ \\
\hline \hline 5768 & 5762 & 5775 & $5760,5763,5766,5769,5776$ & bga \\
\hline 5799 & 5790 & 5810 & $5797,5807,5809,5812$ & $\mathrm{n}$ \\
\hline 5853 & 5850 & 5865 & $5850,5854,5856$ & $\mathrm{n}$ \\
\hline 6197 & $?$ & 6200 & 6196,6199 & $?$ \\
\hline 6204 & 6201 & 6209 & $6199,6203,6205,6212$ & bga \\
\hline 6221 & 6215 & 6230 & $6216,6221,6224,6226,6234$ & bga \\
\hline 6235 & 6232 & 6238 & 6234,6237 & bge \\
\hline 6446 & 6441 & 6449 & 6239,6445 & $?$ \\
\hline 6615 & 6610 & 6621 & 6614 & $\mathrm{n}$ \\
\hline 6711 & 6709 & 6714 & 6709 & $?$ \\
\hline
\end{tabular}

(1) From table 5 in [3], to which I have added $\lambda 6221$.

(2) Estimated peak wavelength of the band. All wavelengths are given in $\AA$ and round up to the nearest integer.

(3) Estimated blue limit of the band.

(4) Estimated red end of the band.

(5) DIBs in the $\left[\lambda_{\text {inf }}, \lambda_{\text {sup }}\right]$ region (from [16]).

(6) 'bg' if the line is in the background, ' $e$ ' if it is a telluric emission line ('a' otherwise), 'n' when no evidence for an atmospheric origin, '?' when the feature is not certain

The list does not intend to be exhaustive. A comparison of table 3 in [3] to the DIB catalog of [16] proves that most, if not all, Red Rectangle bands have possible DIB connections. 
In the $5800 \AA$ region (from $5790 \AA$ to $5950 \AA$ ) a few other DIB/Red Rectangle band associations can be found in $[6,7]$. General remarks on this particularly complex region of the spectrum are given in Sect. 4.2.

At longer wavelengths, six out of the eleven Red Rectangle bands listed in [6], Table $1, \lambda \lambda 6023,6275,6340$, $6460,6537,6578$, are not detected in [3]. I have checked that $\lambda \lambda 6023$ and 6340 do not appear in ESO spectra of the Red Rectangle. The four other lines fall at positions of telluric emission lines [17].

Two other bands in [6] $(\lambda \lambda 6395,6427)$ may correspond to lines in table 3 of [3], but at significantly different peak wavelengths (6399 and $6421 \AA$ ).

\section{DATA ANALYSIS}

In this section and for each Red Rectangle band of Table 2, nebular and background spectra are compared in order to see whether or not the band has an atmospheric origin. If it has a correspondence in the background, 'bg' is reported in the last column of the Table: 'bge' if it is an atmospheric emission line, 'bga' otherwise. 'bga' means that either the band is due to absorption by the atmosphere, or that it exists in the spectrum of HD44179 and belongs to the scattered starlight (in the atmosphere) component of the nebular spectrum (see the following paragraphs and Sect. 5.3). An ' $n$ ' in the last column of the Table means that no straightforward indication of atmospheric origin is found, a '?' that the feature may not be real.

As in [13] background spectra are re-scaled (magnified) so that the amplitude of their variations becomes comparable to those of the nebular spectra, in order to highlight atmospheric absorption features in the spectrum of the nebula.

A telluric emission line is an additive constant, added in the same way to all (background or nebular) spectra, independently of its continuum. It is thus generally easily subtracted during the data reduction process. Since the spectra will be considered before correction of atmospheric absorption, telluric emission lines must appear much larger in the re-scaled background spectra than in the spectra of the nebula.

Absorption by atmospheric molecules affects and modulates the continuum of each spectrum. The resulting fluctuations are, in first approximation, in proportion of the continuum. Therefore, especially if it is weak, the background should be re-scaled prior to a meaningful comparison with the spectrum of the nebula. Atmospheric absorption features will then have similar amplitudes on both spectra, in contrast to telluric emission lines. Re-scaling may

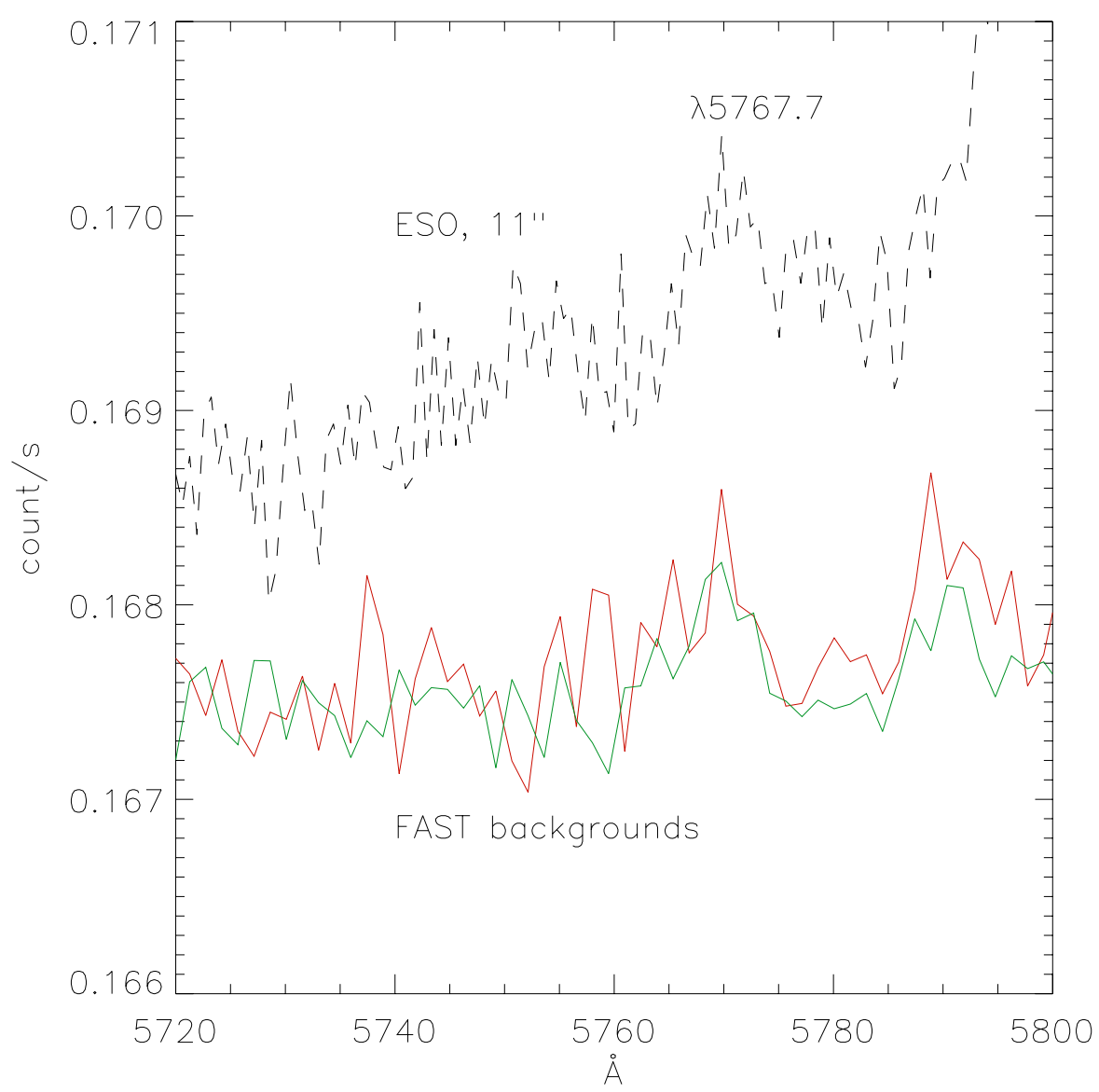

Fig. (1). $\lambda 5768$. ESO observation $11^{\prime \prime}$ from HD44179, average of $s 345$ to $s 364$ in the nebula, and two FAST background spectra (averages of $s 10$ to $s 30$ and $s 90$ to $s 110$ on each side of the slit). FAST backgrounds have been re-scaled for the comparison. 
also highlight small features in the spectrum of HD44179 which may be present in its light scattered in the atmosphere (see Sect. 5).

In [13] I pointed out that the absorption spectrum of a complex molecule comprises 'valleys' and 'peaks', in a similar fashion as an emission spectrum: absorption may thus be easily confused with emission. It also appeared that correction for atmospheric absorption is far less obvious than subtraction of telluric emission lines is.

\section{1. $\lambda \mathbf{5 7 6 8}$}

$\lambda 5768$ is a weak, diffuse band first detected in [7] on the blue edge of the $\lambda 5800$ complex. It begins at $\sim 5764 \AA$, peaks around $5768 \AA$ and has a long red tail that ends between 5775 and $5785 \AA$.

In ESO observations $11^{\prime \prime}$ from HD44179 it is seen from $s 347$ to $\sim s 410,17^{\prime \prime}$ on the sky, and from $\sim s 324$ to $\sim s 410$ (87 pixels, $23^{\prime \prime}$ ) in the observation $6^{\prime \prime}$ from HD44179. It is then too weak to be detected, although re-scaled backgrounds do present a similar feature with minima at the same positions $(\sim 5760$ and $\sim 5785 \AA)$. This feature is well identified and more marked in the FAST backgrounds (Fig.
(1)), and therefore likely related to extinction by the atmosphere (and of 'bga' type).

\section{2. $\lambda 5799$ and $\lambda 5853$}

These two red-degraded Red Rectangle bands were the first to be related to DIBs ( $\lambda 5797$ and $\lambda 5850)$ [4], and are the strongest bands in the $\lambda 5800$ complex. The complex as a whole is proportionally similar at different distances from HD44179 (sect. 5.3 and figures (11) and (12) in [13]). Two bands in the complex, $\lambda \lambda 5912$ and 5937, are found in background spectra.

There is no indication that $\lambda 5799$ and $\lambda 5853$ are of atmospheric origin. The only problem one may find with these bands is that they are detected over $\sim 27^{\prime \prime}$ in ESO observations of the nebula 6 "from HD44179, where the nebula is given to be $9^{\prime \prime}$ wide in [3]. However, Van Winckel et al. give no indication on how they derived the width.

\section{3. $\lambda 6197$}

This feature belongs to the $\lambda 6225$ complex (next subsection). It is not clear to me whether it is real or not. On its

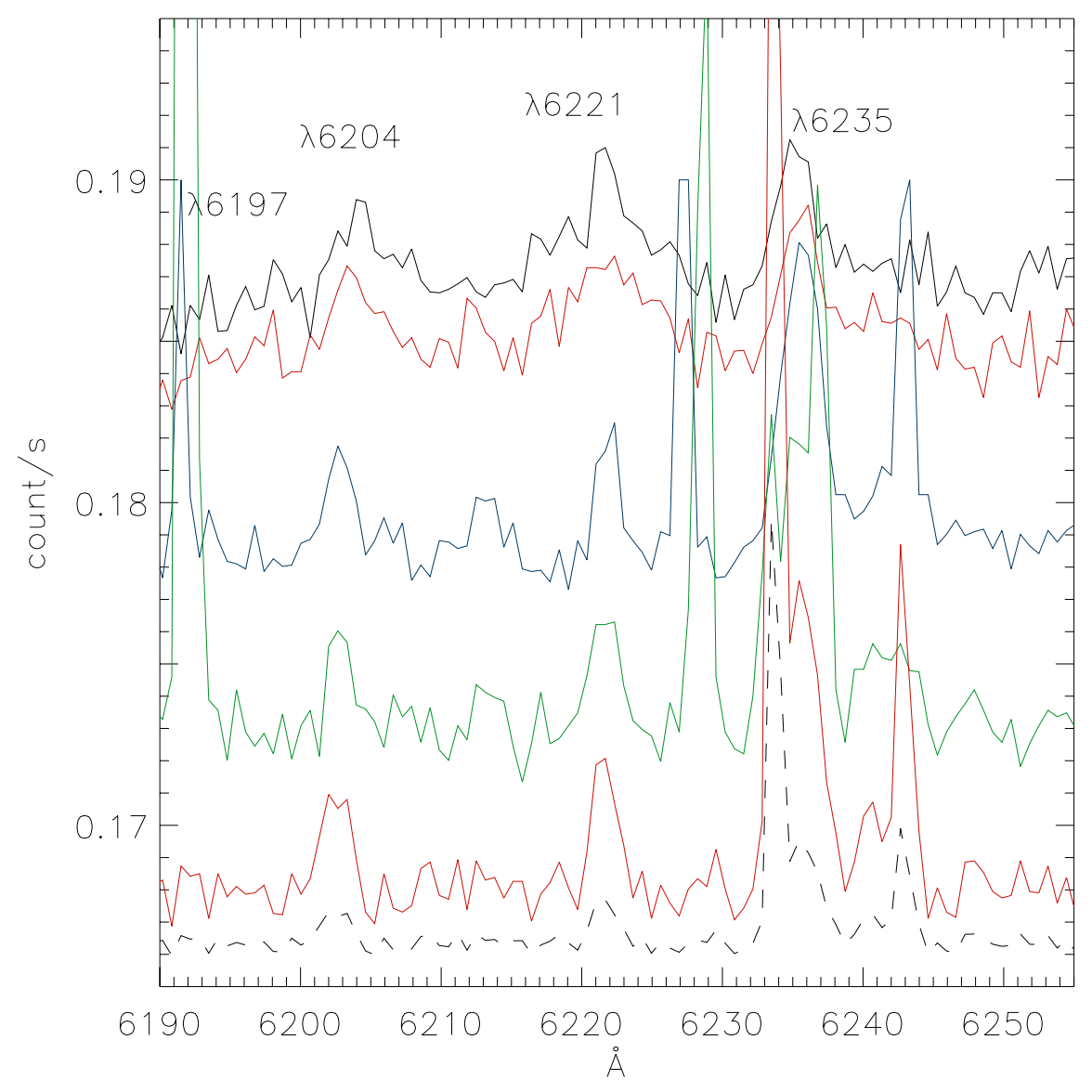

Fig. (2). Fine structure of the $\lambda 6225$ diffuse band. The nebular spectrum (top spectrum), average of $s 355$ to $s 364$ along the slit, in the first ESO observations $11^{\prime \prime}$ north from HD44179, is compared to three re-scaled background spectra from the same observation (from top to bottom, averages of $s 260$ to $s 280$, in blue, $s 460$ to $s 480$, in green, $s 520$ to $s 540$, in red). Background spectra are multiplied by three and arbitrarily shifted for sake of clarity. Top red spectrum is the spectrum of the nebula (average of $s 355$ to $s 364$, no scaling) in the second ESO observation. The bottom dashed spectrum is an example of background ( $s 520$ to $s 540)$ shifted ( +0.004 count/s) but not re-scaled. 
red edge the band is delimited by the minimum on the blue side of Red Rectangle band $\lambda 6204$ (Fig. (2)). Towards the blue, however, it is not possible to separate the feature from the continuum.

\subsection{The $\lambda 6225$ Complex}

$\lambda 6204, \lambda 6221, \lambda 6235$ are sub-structures in Schmidt et al. 's $\lambda 6225$ diffuse band. The spectrum of the nebula $11^{\prime \prime}$ from HD44179 is, in this wavelength region, shown on Fig. (2) (top spectra).

Van Winckel et al. [3] relates red-degraded $\lambda 6204, \lambda 6235$ to DIBs $\lambda 6203$ and $\lambda 6234$ of the $6200 \AA$ DIB complex [14]. DIB $\lambda 6199$ is also close to the blue side of $\lambda 6204$.

$\lambda 6221$ has a symmetric profile, with DIBs $\lambda 6216$ and $\lambda 6226$ on each of its sides.

$\lambda 6204$ and $\lambda 6221$ have clear counterparts in the background (Fig. (2)) of ESO observations. Since they have similar amplitudes on the nebular and re-scaled background spectra, they are not atmospheric emission features (they are of bga type). $\lambda 6235$ also has a correspondence in the background (see also middle right plot of figure (7) in [13]). The line is much larger on the re-scaled background spectra than on the nebular ones, which indicates it is an atmospheric emission line. It does fall at the exact position of an emission line identified in the La Silla-Paranal night sky spectrum (figure (23) in [17]).

Fig. (3) shows that subtraction of the background largely removes $\lambda 6235$ but has no significant effect on $\lambda \lambda 6204$, 6221. These bga features still remain in the Van Winckel et al. [3] spectra after complete data-reduction; they are thus not corrected by standard routines.

These observations confirm the finding in [13], that the structure of the nebula's spectrum in the $6200 \AA$ region is determined by atmospheric extinction.

\section{5. $\lambda 6446$}

$\lambda 6446$ is a small feature which appears on the average of a few spectra ( $s 355$ to $s 364$, Fig. (4)) within the nebula, in the first ESO observation $11^{\prime \prime}$ from HD44179. However it cannot be distinguished from the continuum in the individual

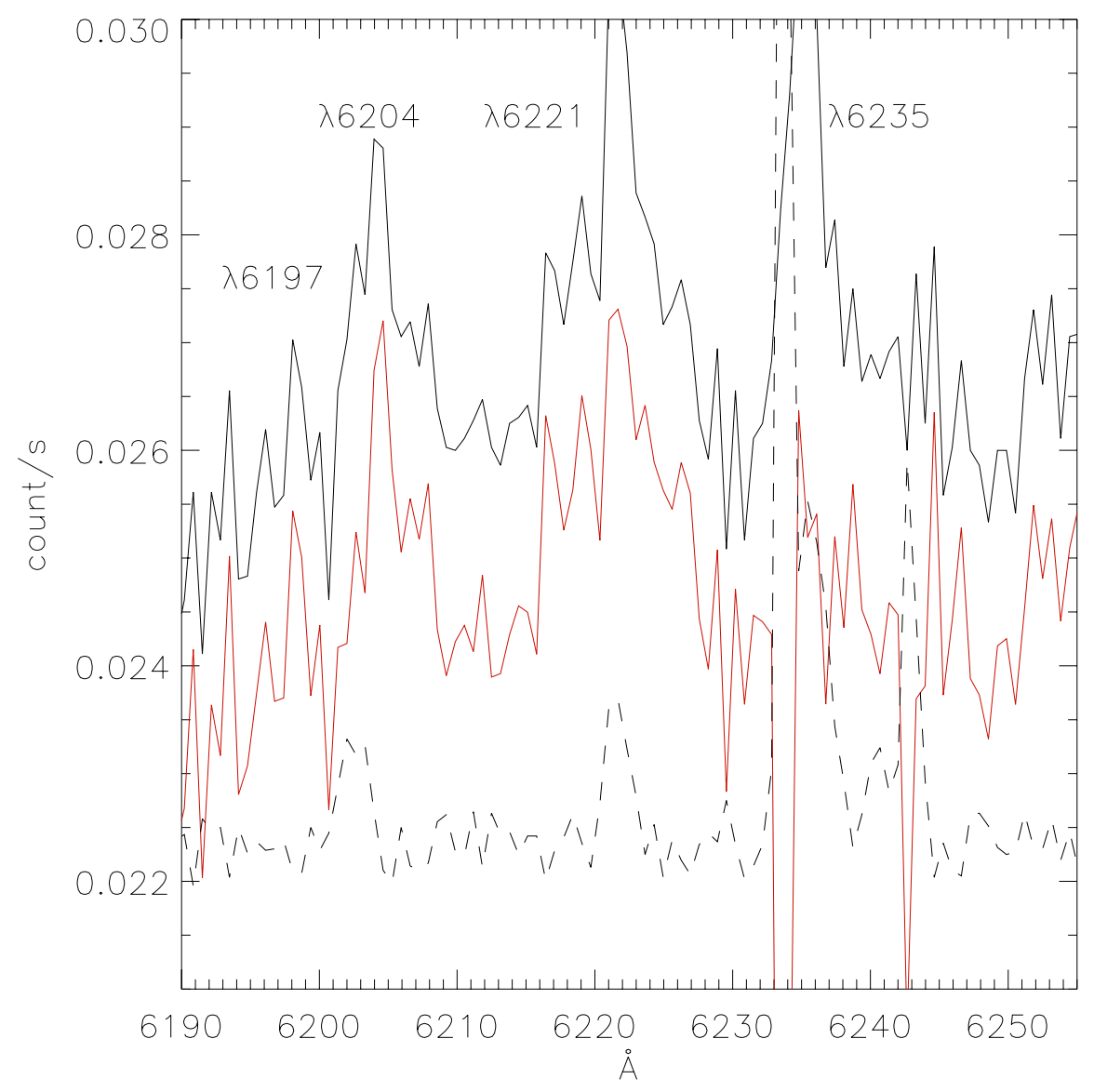

Fig. (3). Fine structure of the $\lambda 6225$ diffuse band. Top spectrum is, as in Fig. (2), a nebular spectrum (average of $s 355$ to $s 364$ ), in the first ESO observations $11^{\prime \prime}$ north from HD44179, shifted by -0.1605 count/s. Bottom dashed spectrum is a background spectrum (average of $s 520$ to $s 540$ offset by -0.14 count/s). Middle red spectrum is the spectrum of the nebula minus the background. Structures in the background clearly remain in the spectrum of the nebula after subtraction of the background, and will not be removed by normalization by a standard star. In general standard data-reduction routines are inefficient in correcting these effects of atmospheric extinction. 


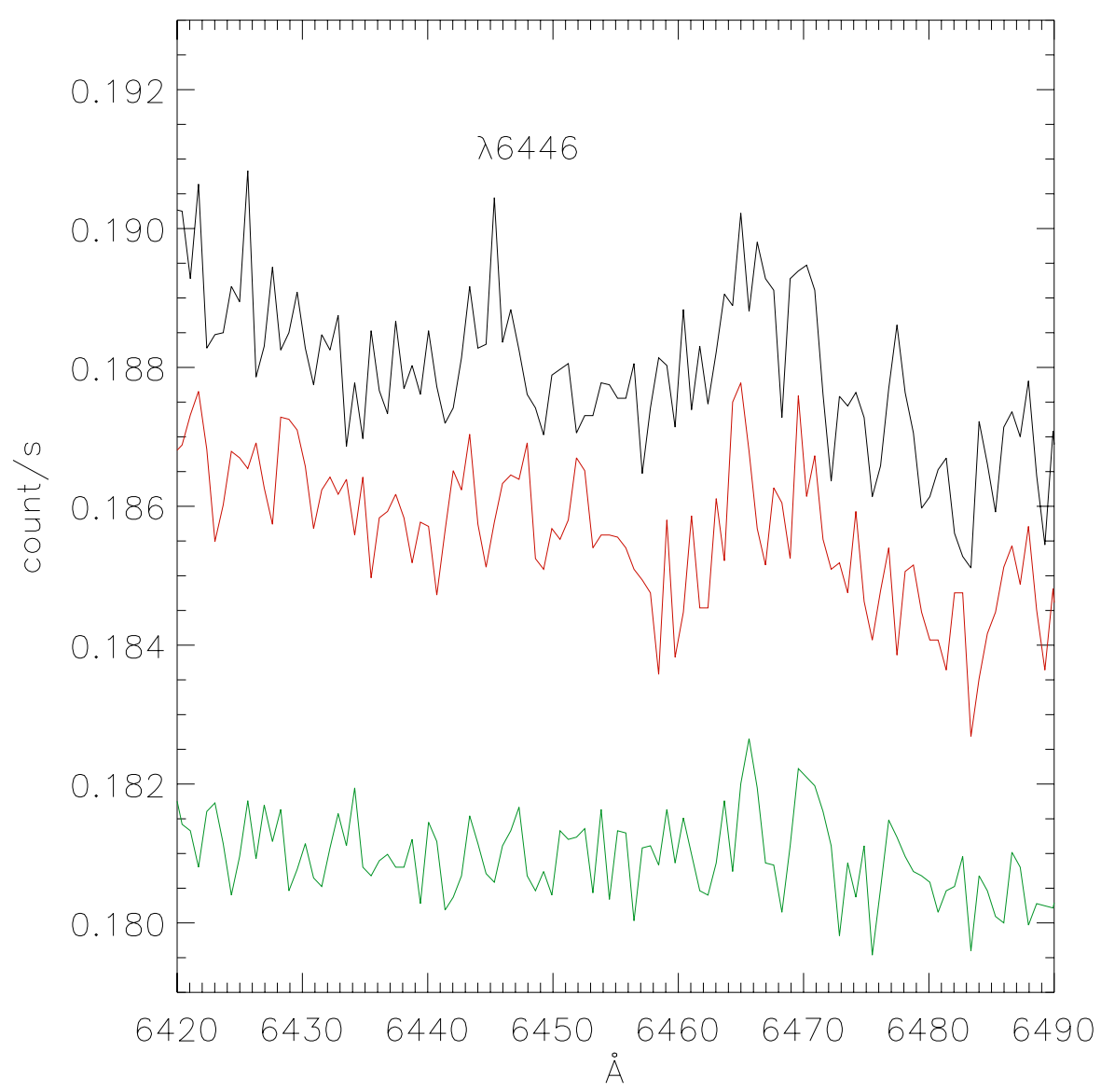

Fig. (4). $\lambda 6446$. Top spectrum is the average of $s 355$ to $s 364$ of the first ESO observation of the nebula, $11^{\prime \prime}$ from HD44179. In green, the average of $s 340$ to $s 348$ (shifted by +0.01 ), same observation. Red spectrum is the average of $s 353$ to $s 361$ in the second, similar, ESO observation. All three spectra are in the nebula. The group of lines close to $6465 \AA$ are night sky emission lines [17] also present in background spectra (not represented here).

spectra of this observation, or in other averaged spectra (e.g., from $s 340$ to $s 348$, Fig. (3)). It is also absent from the average spectrum of the second identical ESO observation. It may therefore be an artifact.

\section{6. $\lambda 6615$}

$\lambda 6615$ is a more precise determination of Schmidt et al.'s $\lambda 6617$ band. In ESO observations $11^{\prime \prime}$ from HD44179 it is $10 \AA$ wide (from $\sim 6610$ to $\sim 6620 \AA$ ).

It is observed on a slightly larger extent than found for the nebula ([13], sect. 5.2), and the background seems to reproduce its fine structure. These observations are however at the limit of the error margin, thus can not be used to discuss rigorously the origin of the band.

\section{7. $\lambda 6711$}

As for $\lambda 6197$ and $\lambda 6446$ the existence of this last feature, from an analysis of individual spectra in ESO observations $11^{\prime \prime}$ from HD44179, does not seem to me obvious.

There is a spike (Fig. (5)) in a few spectra (from $s 349$ to $s 358$ ) of the first ESO observation $11^{\prime \prime}$ from HD44179. This spike, probably a cosmic ray, is absent from all other spectra as well as in the second identical observation of the nebula (Fig. (5)). There may be a slight enhancement of the continuum around $6711 \AA$ on Fig. (5) but not significant enough to be considered as evidence, as it is done in [3], for an emision line.

\section{DISCUSSION}

\subsection{The Red Rectangle Bands}

Reserves should be made on the reality of three ( $\lambda \lambda 6197$, $6445,6711)$ out of the nine Van Winckel et al. bands associated to DIBs. $\lambda \lambda 6445$ and 6711 seem to be accidental artifacts in a few spectra of one of the observations $11^{\prime \prime}$ from HD44179. $\lambda 6197$ may be caused by the minimum on $\lambda 6204$ 's blue side.

Four other bands $(\lambda \lambda 5768,6204,6221,6235)$ have a correspondence in the background, which I believe is not accidental. One $(\lambda 6235)$ is a telluric emission line listed in [17]. The three others (two of which belong to the $\lambda 6225$ complex), unless they are related to light from HD44179 scattered in the atmosphere (Sect. 5.3), must be due to absorption in the atmosphere. 


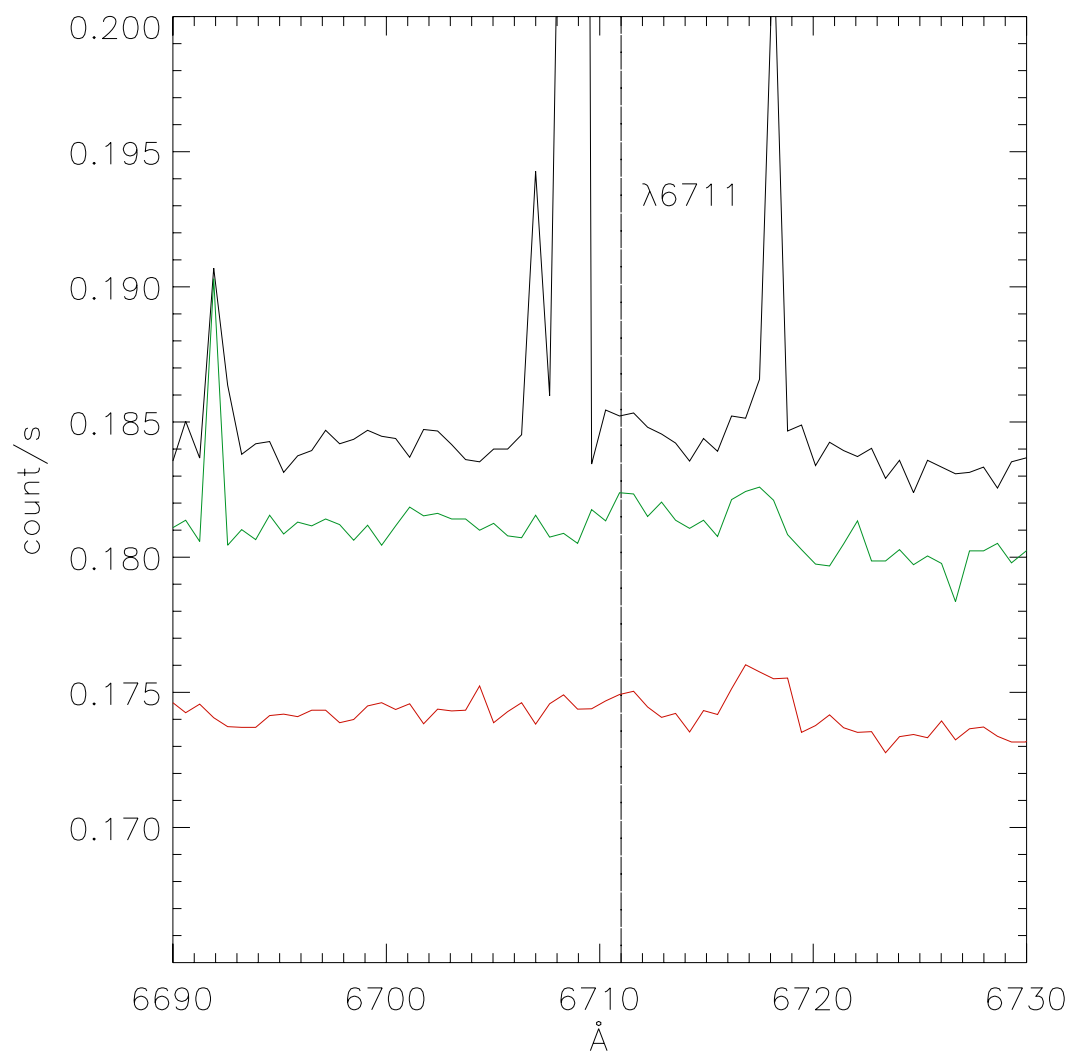

Fig. (5). $\lambda 6711$. In black, average spectrum of $s 349$ to $s 358$, in the nebula, first ESO observation, $11^{\prime \prime}$ from HD 44179 . Green is the average of $s 359$ to $s 370$, still in the nebula, same observation. Red spectrum is the average of $s 340$ to $s 360$ (in the nebula) in the second, identical, ESO exposure. The bump centered at $6717 \AA$ in the two later spectra is an emission sky line listed in [17].

Sect. 3 has shown that similar bias exist in other datasets: over $50 \%$ of the bands found in [6] outside the $5800 \AA$ region are not detected in [3], and are probably either artifacts or due to the atmosphere.

The three remaining bands in Table $\mathbf{2}$, among which two are in the $\lambda 5800$ complex, may arise in the nebula, although this remains to be proved.

This study thus confirms the importance of the atmosphere, especially in the $6200 \AA$ region, in the observed spectrum of the Red Rectangle.

\subsection{The Relationship Between DIBs and Red Rectangle Bands}

DIBs may now be found in any part of the visible spectrum, and it will in general be easy to find DIBs in the vicinity of each Red Rectangle band (see fourth column of Table 2).

On the one hand, the uncertainty on the Red Rectangle bands, their relatively low (compared to $\mathrm{S} / \mathrm{N}$ ratios achieved in DIB observations) level of detection, show how careful one must be when associating DIBs and Red Rectangle bands.

On the other hand DIB/Red Rectangle band associations do not rely only on wavelength coincidences. Other criteria, as the importance of the bands in each spectrum, have to be taken into account. In the $5800 \AA$ region for instance, it would indeed be remarkable that two sets of prominent features, in the spectra of reddened stars and in the spectrum of a nebula, can coincide and be independent.

There might thus be less DIB/Red Rectangle band true associations than previously though. In addition, the difficulties raised by the relative positions of these DIBs and Red Rectangle bands on the spectrum, and the objections in [10], could be overcome if the Red Rectangle spectrum was an absorption spectrum: it is then the valleys on the steep sides of the Red Rectangle bands in the nebular spectra which have to coincide with DIBs, as observed.

\subsection{Red Rectangle Bands in Background Spectra}

Red Rectangle bands $\lambda 5768$ and $\lambda \lambda 6204,6221,6235$ in the $\lambda 6225$ complex, are found in background spectra.

The presence of DIBs $\lambda \lambda 6234$ and 6237 on the sides of $\lambda 6235$ may be a coincidence since the band is at the exact position of a telluric emission line.

The presence in the background of the other bands and a relation to DIBs is more difficult to understand, but may be justified by either of the following possibilities.

These Red Rectangle bands can be due to an incomplete removal of atmospheric absorption [13]. If they are related to DIBs, it will necessarily be concluded that the corresponding DIBs are also atmospheric absorption bands, and have not been removed during the data reduction process. Next section shows that this hypothesis, as unexpected as it can be, can not be ruled out. 
This hypothesis could explain the complexity of the DIB spectrum, but would also mean that some DIBs need to have, in addition to an interstellar cloud, the atmosphere on the line of sight. Should it be the case, the relative roles of the interstellar medium and of the atmosphere, in the formation of these DIBs, need to be elucidated. It cannot concern all DIBs since some are detected with high redshifts in other galaxies (for instance [19] for the Magellanic Clouds, [20] for NGC 1448).

Another possibility is that DIBs, although they are not observed toward HD44179, exist at a low level in the spectrum of the star, and are detected in the light from HD44179 scattered by the nebula and/or the atmosphere. This hypothesis would justify that the observed spectrum of the nebula is an absorption spectrum, and its relationship to the DIBs.

Alternatively, Red Rectangle bands due to the atmosphere may not be related to DIBs.

Space observations of the Red Rectangle nebula and of reddened stars with the Hubble Space Telescope (HST) will provide the most straightforward answer to these hypotheses.

\subsection{The $\lambda 6200$ DIB Complex}

As the $5800 \AA$ region, the $6200 \AA$ region (from $\sim 6150$ to $\sim 6250 \AA$ ) concentrates many features whose existence is doubtless, and coincide in the DIB and Red Rectangle spectra.

In [13] the diffuse $\lambda 6225$ Schmidt et al. Red Rectangle band was attributed to atmospheric absorption, and more specifically to absorption by $\mathrm{O}_{4}$ on its red side (sect. 6.3 and figure (14) in [13]). Sect. 4.4 indicates that its fine structure may also be due to the atmosphere. A link between the Red Rectangle band and the $\lambda 6200$ DIB complex would thus imply that the latter results from atmospheric absorption.

Fig. (6). compares the raw spectrum (before corrections for atmospheric extinction, radial velocity and normalization to the continuum) of $\mathrm{BD}+40^{\circ} 4220$ (green spectrum of the figure) observed by G. Galazutdinov, and A. Jenouvrier's laboratory spectrum of $\mathrm{NO}_{2}$ (in red), one among the most important and complex molecules in the atmosphere.

There are evident similarities between the spectra. Low frequency variations are common to both. The broad DIB $\lambda 6177$, reported in earlier papers $[14,18]$ and seen here with

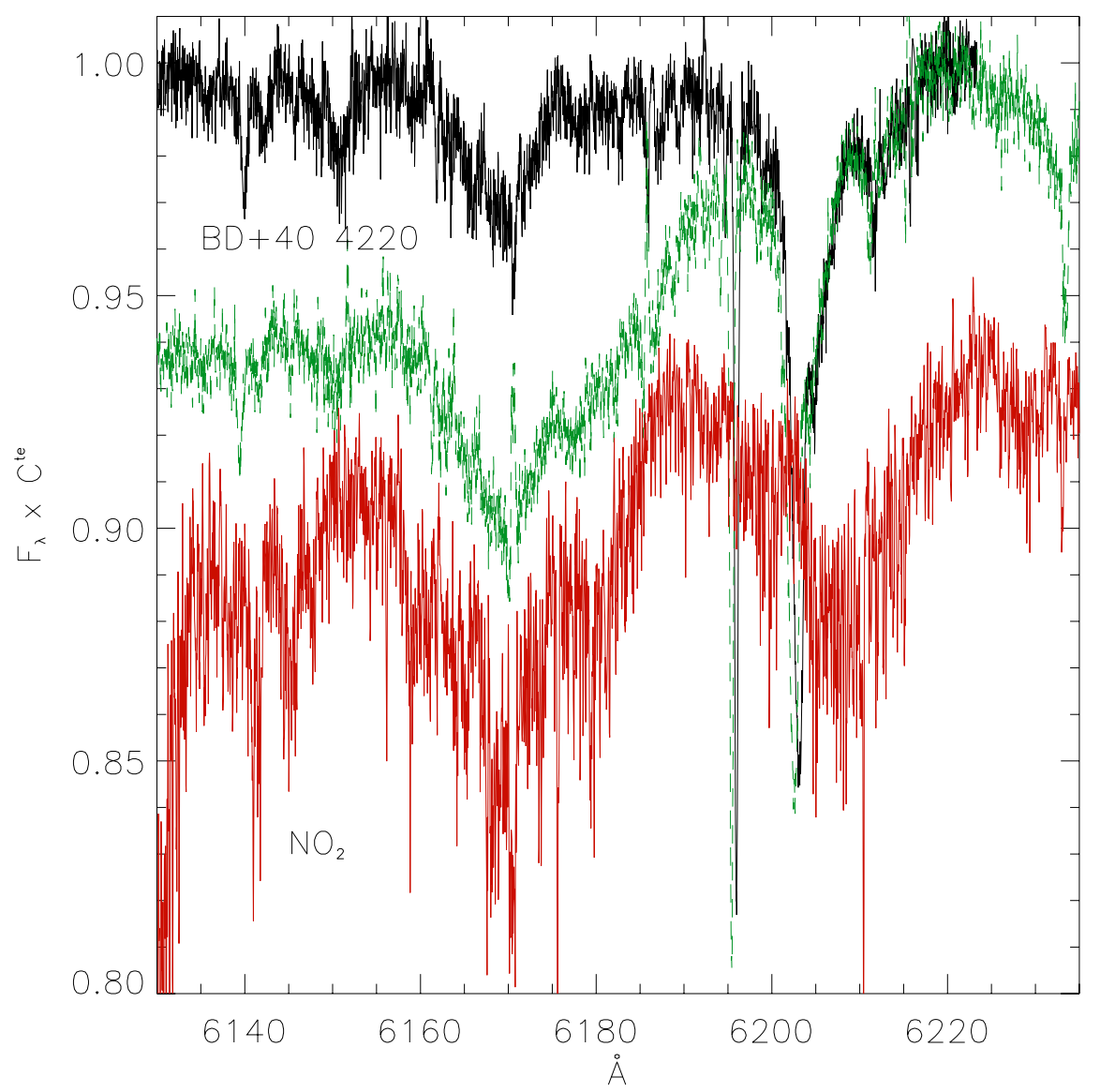

Fig. (6). The raw (before correction for atmospheric extinction) spectrum of BD $+40^{\circ} 4220$ (resolution $=0.03 \AA$ ) in green (courtesy of G. Galazutdinov) is compared to the absorption spectrum of $\mathrm{NO}_{2}$ (in red, resolution $=0.01 \AA$ ) in the $6200 \AA$ region. The top black spectrum is $\mathrm{BD}+40^{\circ} 4220$ 's after data reduction (figure (14) in [16]). 
much better resolution, is a clear, recognizable feature of the spectrum of $\mathrm{NO}_{2}$. Narrower DIBs in the [16] catalog, $\lambda 6139.94, \lambda 6145.65 \quad \lambda 6158.54, \lambda 6167.84, \lambda 6220.82$, $\lambda 6223.56, \lambda 6226.3$ also have counterparts in the spectrum of $\mathrm{NO}_{2}$. Differences of position of the features' minima between the spectra are insignificant, considering the complexity of $\mathrm{NO}_{2}$ absorption (which is not resolved in either observations), and the different observational conditions of the two spectra.

The spectra also have differences, mainly the two sharp, strong absorption lines (DIBs) at 6196 and $6203 \AA$ (on the blue side of Red Rectangle band 26204 ), noticeably absent from the $\mathrm{NO}_{2}$ spectrum, and two small absorption features at $6151 \AA$ and $6186 \AA$.

I found no straightforward identification for these lines. DIBs $\lambda \lambda 6196$ and 6204 were detected in NGC1448 [20] with a redshift of $\sim 24 \AA(\sim 1160 \mathrm{~km} / \mathrm{s})$, which implies an interstellar origin.

On the right hand side of the plot, the structure between 6210 and $6230 \AA$ in the spectrum of $\mathrm{NO}_{2}$ (in the spectrum of $\mathrm{BD}+40^{\circ} 4220$ as well) coincides with Red Rectangle band $\lambda 6221$ (Fig. (2)). Attribution of the diffuse $\lambda 6225 \mathrm{Red}$ Rectangle band to $\mathrm{O}_{4}$ 's absorption on its red side, and of part of its sub-structure to $\mathrm{NO}_{2}$, would mean that several atmospheric molecules may contribute to the Red Rectangle bands in a given wavelength region.

The DIB spectrum (in black, see also figures (14) and (15) in [16]), obtained after data reduction, does not differ much from the raw spectrum except that the continuum is normalized and redshifted (by $1 \AA \sim 30 \mathrm{~km} / \mathrm{s}$ ) to correct for the radial velocity of the interstellar cloud on the line of sight and for the motion of the earth, assuming that absorption is all interstellar. Normalization of the continuum has rubbed part of the similarity with the $\mathrm{NO}_{2}$ spectrum, especially the steep decrease between $6170 \AA$ and $6190 \AA$. The broad DIB absorption centered close to $6170 \AA$, as well as smaller features are still present in the DIB spectrum.

It is evident that, if $\mathrm{NO}_{2}$ determines the structure of the raw spectrum in this wavelength region, the data-reduction process did not remove all effects of atmospheric absorption. Some absorption bands which have been attributed to absorption in the interstellar cloud, are due to atmospheric $\mathrm{NO}_{2}$ (possibly also to other molecules in the atmosphere).

\section{CONCLUSION}

This study has questioned the origin of Red Rectangle bands which have been considered as DIBs in emission. The presence of some bands in background spectra questions the nature of the Red Rectangle spectrum (emission or absorption, interstellar or due to the atmosphere?), as it is observed from earth, the Red Rectangle band/DIB relationships, and the nature of some DIBs.

I have also presented observations which show the remarkable similarity between the DIB spectrum of a reddened star and a laboratory spectrum of $\mathrm{NO}_{2}$, in the $[6100,6240] \AA$ wavelength range. If part of DIB complex $\lambda 6200$ is indeed due to atmospheric absorption, its observation in the spectrum of reddened stars needs, in addition to an interstellar cloud, to have the atmosphere on the line of sight. In this case, the respective roles of the interstellar cloud and the atmosphere, in the observation of this DIB, need to be elucidated.

Observed DIBs and Red Rectangle spectra may therefore be caused by different kinds of extinction phenomena, interstellar but also atmospheric, which render the interpretation of these ground-based observations particularly difficult and complex. To separate atmospheric and interstellar effects in the DIBs and Red Rectangle spectra, the easiest and most reliable way would be to obtain spectra from outside the atmosphere, with the HST for instance (to this date, no such observational program has been performed).

\section{ACKNOWLEDGMENTS}

I am highly indebted to G. Galazutdinov and to A. Jenouvrier for allowing me to use their observations of $\mathrm{BD}+40^{\circ} 4220$ and $\mathrm{NO}_{2}$ in this publication. I also wish to thank anonymous referees for their careful reading of the manuscript and useful comments.

This research has used observations made with ESO NTT Telescope at La Silla-Paranal Observatory under programme ID $60 . c-0473$.

\section{REFERENCES}

[1] Schmidt GD, Cohen M, Margon B. Discovery of optical molecular emission from the bipolar nebula surrounding HD 44179. Astrophys J 1980; 239: L133-8.

[2] Warren-Smith RT, Scarrott SM, Murdin P. Peculiar optical spectrum of the Red Rectangle. Nature 1981; 292: 317-9.

[3] Van Winckel H, Cohen M, Gull TR. The ERE of the "Red Rectangle" revisited. Astron Astrophys 2002; 390: 147-54.

[4] Fossey SJ. thesis, Univ. of London, 1990.

[5] Sarre PJ. Diffuse bands in emission. Nature 1991; 351: 356.

[6] Scarrott SM, Watkin S, Miles JR, Sarre PJ. Evidence for a link between the more prominent optical emission bands in the Red Rectangle and some of the diffuse instellar absorption. Mon Not R Astron Soc 1992; 255: 11-6.

[7] Sarre PJ, Miles JR, Scarrott SM. Molecular diffuse interstellar band carriers in the Red Rectangle. Science 1995; 269: 674-76.

[8] Schmidt GD, Witt AN. X marks the SPOT - Distribution and excitation of unidentified molecules in the Red Rectangle. Astrophys J 1991; 383: 698-704.

[9] Herbig GH. The diffuse interstellar bands. Annu Rev Astron Astrophys 1995; 33: 19-74.

[10] Glinski RJ, Anderson CM. On the Red Rectangle optical emission bands. Mon Not R Astron Soc 2002; 332: L17-L22.

[11] Hobbs LM, York DG, Snow TP, et al. A catalog of diffuse interstellar bands in the spectrum of HD 204827. Astrophys J 2008; 680: 1256-70.

[12] Zagury F. An Analysis of Spectra in the Red Rectangle Nebula. J Astrophys Astron 2005; 26: 395-420.

[13] Zagury F. Analysis of the Schmidt, Cohen \& Margon (1980) features in the Red Rectangle nebula. Open Astr J 2009; 2: 26-38.

[14] Chlewicki G, de Groot MS, van der Zwet GP, Greenberg JM, Alvarez PP, Mampso A. Correlation of broad and narrow diffuse band features evidence for molecular carriers. Astron Astrophys 1987; 173: 131-6.

[15] Fabricant D, Cheimetz P, Caldwell N, Geary J. The FAST Spectrograph for the tillinghast telescope. Publ Astron Soc Pac 1998; 110: 79-85.

[16] Galazutdinov GA, Musaev FA, Krelowski J, Walker GAH. Narrow diffuse interstellar bands: A survey with precise wavelengths. Publ Astron Soc Pac 2000; 112: 648-90.

[17] Hanuschik RW. A flux-calibrated, high-resolution atlas of optical sky emission from UVES. Astron Astrophys 2003; 407: 1157-64. 
[18] Le Bertre T, Lequeux J. Diffuse absorption bands in the spectra of mass losing objects. Astron Astrophys 1993; 274: 909-14.

[19] Ehrenfreund P, Cami J, Jim enez-Vicente J, et al. Detection of diffuse interstellar bands in the magellanic clouds. Astrophys $\mathrm{J}$ 2002; 576: L117-20.
[20] Sollerman J, Cox N, Jakobson P, et al. Diffuse interstellar bands in NGC 1448. Astron Astrophys 2005; 429: 559-67.

Received: December 23, 2008

Revised: February 23, 2009

Accepted: February 27, 2009

(c) Frédéric Zagury; Licensee Bentham Open.

This is an open access article licensed under the terms of the Creative Commons Attribution Non-Commercial License (http://creativecommons.org/licenses/bync/3.0/), which permits unrestricted, non-commercial use, distribution and reproduction in any medium, provided the work is properly cited. 